\title{
SEMICONVEX SPACES II
}

\author{
by S. O. IYAHEN \\ (Received 11 March, 1968; revised 23 December, 1968)
}

1. Introduction. One of the concepts introduced in [2] is that of a hyperbornological space, an idea which effectively replaces that of a bornological space when semiconvex spaces are being considered. In Section 2 of the present paper, it is shown how the topology of such a space may be described in terms of bounded pseudometrices. This is used in Section 3 to tackle the problem of when a product of separated hyperbornological spaces has the same property. It is shown that, as in the classical case of bornological spaces, this problem is equivalent to one in measure theory.

Throughout, our linear spaces shall be over the real field, denoted by $R$. We shall shorten "linear topological space" to l.t.s., "locally convex l.t.s." to locally convex space, and " neighbourhood of the origin" to neighbourhood.

2. Pseudometrics on hyperbornological spaces. Let $E$ be a linear space (over the reals). If $\lambda>0$, a subset $A$ of $E$ is said to be $\lambda$-convex if $A+A \subseteq \lambda A$. A subset of $E$ which is $\mu$-convex for some $\mu>0$ is called a semiconvex set. If $u$ is a linear topology on $E$, the l.t.s. $(E, u)$ is called a semiconvex space if it has a base of neighbourhoods of the origin consisting of balanced semiconvex sets.

According to S. Simons ([3], p. 170), a function $p$ from a linear space $E$ into the nonnegative reals (denoted by $\left.R^{+}\right)$is called a $k$-pseudometric $(0<k \leqq 1)$ if

(i) there is a point $x$ in $E$ such that $p(x) \neq 0$,

(ii) $p(x+y) \leqq p(x)+p(y)$ for all points $x, y$ in $E$, and

(iii) $p(\lambda x)=|\lambda|^{k} p(x)$ for each point $x$ in $E$ and $\lambda$ in $R$.

As observed in Section 2 of [2], the notion of a semiconvex space coincides with that of an upper bound space, due to S. Simons ([3], Definition 5). Thus an 1.t.s. $(E, u)$ (where $u$ is not the trivial topology) is a semiconvex space if and only if there is a family $\left\{p_{\alpha}: \alpha \in \Phi\right\}$ of $(u-$ continuous) $k_{\alpha}$-pseudometrics on $E$ such that the family of sets $\left\{x: p_{\alpha}(x)<1\right\}$ form a base of neighbourhoods of the origin in $(E, u)$.

A semiconvex space $E$ is said to be hyperbornological if every bounded linear map from $E$ into a semiconvex space is continuous (see [2], Section 3). This is equivalent to the stipulation that every balanced semiconvex bornivorous proper subset of $E$ is a neighbourhood.

A $k$-pseudometric on an l.t.s. $E$ is said to be bounded if it maps each bounded subset of $E$ into a bounded set of non-negative real numbers.

PROPOSIrION 1. A semiconvex space $E$ is hyperbornological if and only if every bounded $k$-pseudometric $(0<k \leqq 1)$ on $E$ is continuous.

Proof. Suppose that $E$ is hyperbornological, and that, for some $k$ such that $0<k \leqq 1$, $p$ is a bounded $k$-pseudometric on $E$. Let $U=\{x: p(x) \leqq 1\}$. The set $U$ is balanced and, being 
$2^{1 / k}$-convex, is semiconvex. Also, $U$ is bornivorous. For if $A$ is a bounded subset of $E$, there is a positive real number $r$ such that $p(x) \leqq r$ for all $x$ in $A$, since $p$ is bounded. This implies that $A \subseteq r^{1 / k} U$, and thus $U$ is bornivorous. As $E$ is hyperbornological, $U$ is a neighbourhood in $E$, and thus $p$ is continuous.

Now suppose that $U$ is a balanced semiconvex bornivorous proper subset of $E$. For some $\mu \geqq 3, U+U+U \subseteq \mu U$. Write $k$ for $\log _{\mu} 2$. For each $x$ in $E$, let

$$
d(x)=\inf \left(\lambda^{k}: \lambda \in R^{+}, x \in \lambda U\right),
$$

and $f(x)=\inf \left(\sum_{i} d\left(x_{i}\right)\right)$ with $\left(x_{i}\right)$ ranging over all finite subsets of $E$ such that $\sum_{i} x_{i}=x$. As shown in Lemma 1 of [3], $f$ is a $k$-pseudometric on $E$ and for each $x$ in $E, \frac{1}{2} d(x) \leqq f(x) \leqq d(x)$. Now, $f$ is bounded. For, if $A$ is a bounded subset of $E$, then there is a positive real number $r$ such that $A \subseteq r U$, since $U$ is bornivorous. Thus for each $x$ in $A, f(x) \leqq d(x) \leqq r^{k}$, and $f$ is bounded. If every bounded $k$-pseudometric on $E$ is continuous, $U$ must be a neighbourhood in $E$, since $\left\{x: f(x)<\frac{1}{2}\right\} \subseteq U$.

3. Products of hyperbornological spaces. Every metrizable semiconvex space is hyperbornological. In particular, $R$ has the property. It is pointed out after Theorem 3.5 of [2] that a product $\times_{\gamma \in I} E_{y}$ of separated arbitrary hyperbornological spaces is hyperbornological if (and only if of course) $R^{I}$ is hyperbornological. We shall now adapt the approach in [4] to find out when $R^{I}$ is hyperbornological. As our arguments are similar to these in [4], all we shall do is to point out the modifications needed in [4] to get our result, and in the process we shall stick closely to the notation used there.

An ultrafilter which is closed under countable intersections is called a $\delta$-ultrafilter; $\delta$-ultrafilters are also called Mackey-Ulam ultrafilters (see [1], pp. 279-281). A $\delta$-ultrafilter on a set $I$ is said to be trivial if it is the collection of all subsets of $I$ which contain a fixed element of $I$. As observed in Section 1 of [4], the existence of a non-trivial $\delta$-ultarfilter on $I$ is equivalent to the existence of a two valued measure defined on all subsets of $I$ such that the measure of every singleton set is zero. It is still unknown if such a measure exists. S. Ulam in [5] showed that a set on which such a measure exists must be very large and must in fact have a cardinal which is strongly inaccessible.

We generalize the concept of a magnitude (see Definition 4 of [4]). We say that $m$ is a $k$-hypermagnitude $(0<k \leqq 1)$ on $R^{I}$ if

(a) $m$ is a $k$-pseudometric on $R^{I}$,

(b) $0 \leqq f \leqq g \Rightarrow m(f) \leqq m(g)\left(f, g \in R^{I}\right)$, and

(c) $m(f)=m(|f|)\left(f \in R^{I}\right)$.

It is not difficult to show that, if $m$ is a magnitude on $R^{I}$, then for any $k$ such that $0<k \leqq 1$, the function $m_{k}(f)=(m(f))^{k}, f \in R^{I}$, is a $k$-hypermagnitude on $R^{I}$.

Lemmas 1, 2, 3 of [4] are true if "magnitude" is replaced by " $k$-hypermagnitude". The arguments in [4] also work in this case. Also, with a method of proof similar to that in Lemma 13 of [4], one shows that any $k$-hypermagnitude on $R^{I}$ is bounded, and that every bounded $k$-pseudometric on $R^{I}$ is dominated by some $k$-hypermagnitude. 
From this and Proposition 1 above, we deduce that $R^{I}$ is hyperbornological if and only if every $k$-hypermagnitude on $R^{I}$ is continuous.

If $m$ is a $k$-hypermagnitude on $R^{I}$ and $J \subseteq I, m$ is said to be non-zero on $J$ if there is an element $f$ of $R^{I}$ with support contained in $J$ such that $m(f)>0$.

As Lemmas 1 and 2 of [4] are true if " magnitude " is replaced by " $k$-hypermagnitude ", the set $\mu$ of all subsets of $I$ on which a $k$-hypermagnitude $m$ is non-zero is a magnum (for the definition of magnum, see [4]). Hence, by Theorem 3 of [4], $\mu$ is a finite union of $\delta$-ultrafilters.

Any $f$ in $R^{l}$ can be extended to the set of all $\delta$-ultrafilters $\mathscr{F}$ on $I$; since $f(\mathscr{F})$ is a trivial $\delta$-ultrafilter base on $R$, it contains a one point set $(r)$ and Simons [4] puts $f(\mathscr{F})=r=\lim _{\mathscr{F}} f$. If

$$
m_{k}(f)=\max \left(|f(\mathscr{F})|^{k}: \mathscr{F} \subseteq \mu, \mathscr{F} \text { a } \delta \text {-ultrafilter }\right),
$$

then $m_{k}$ is a $k$-hypermagnitude lying between positive multiples of $m$, and the corollary of Lemma 12 and its proof can be modified to show that $m_{k}$ (and hence $m$ ) is continuous if and only if $\mu$ consists of trivial $\delta$-ultrafilters.

Thus $R^{I}$ is hyperbornological (every $k$-hypermagnitude on $R^{I}$ is continuous) if and only if every $\delta$-ultrafilter on $I$ is trivial. We deduce in particular that if the cardinal of an index set $\Phi$ is not strongly inaccessible, then a product $x_{\gamma \in \Phi} E_{y}$ of separated hyperbornological spaces is hyperbornological.

The author wishes to thank the referee for his suggestions.

\section{REFERENCES}

1. A. Grothendieck, Espaces vectoriels topologiques (Sao Paulo, 1958).

2. S. O. Iyahen, Semiconvex spaces, Glasgow Math. J. 9 (1968), 111-118. 169-180.

3. S. Simons, Boundedness in linear topological spaces, Trans. Amer. Math. Soc. 113 (1964), $461-473$.

4. S. Simons, The bornological topology associated with $R^{I}$, J. London Math. Soc. 36 (1961),

5. S. Ulam, Zur Masstheorie der allgemeinen Mengenlehre, Fund. Math. 16 (1930), 140-150.

UNIVERSITY OF IBADAN

IBADAN, NIGERIA 